\title{
The use of an uncovered stent in type A aortic dissection during open aortic surgery
}

\author{
Leopold Rupprecht, MD, ${ }^{\mathrm{a}}$ Piotr Kasprzak, $\mathrm{PhD},{ }^{\mathrm{b}}$ Christof Schmid, $\mathrm{PhD},{ }^{\mathrm{a}}$ Reinhard Kobuch, MD, ${ }^{\mathrm{a}}$ and \\ Karin Pfister, PhD, ${ }^{\text {b }}$ Regensburg, Germany
}

\footnotetext{
From the Departments of a Cardiothoracic Surgery and ${ }^{b}$ Vascular Surgery, University Medical Center, Regensburg, Germany.

Disclosures: Authors have nothing to disclose with regard to commercial support.

Received for publication April 20, 2018; revisions received June 1, 2018; accepted for publication June 7, 2018 available ahead of print July 24, 2018

Address for reprints: Leopold Rupprecht, MD, Department of Cardiothoracic Surgery, University Medical Center, Franz-Josef-Strauss-Allee 11, 93053 Regensburg, Germany (E-mail: leopold.rupprecht@ukr.de).

J Thorac Cardiovasc Surg 2018;156:e199-200 $0022-5223 / \$ 36.00$

Copyright $(2018$ by The American Association for Thoracic Surgery https://doi.org/10.1016/j.jtcvs.2018.06.026
}

The optimal surgical techniques for the treatment of patients with type A aortic dissection are still under debate. Mere replacement of the ascending aorta and optionally also the proximal arch is the usual minimum surgical option, yielding low perioperative mortality. About $15 \%$ of patients have long-term sequelae develop, mainly falselumen growth, as a result of persistent dissection within the aortic arch. ${ }^{1}$ More extensive surgical procedures with complete replacement of the aortic arch, especially the frozen elephant trunk technique, prevent this complication; but they are technically very demanding and place a much larger burden on the patient.

We report the case of a patient in whom we used an alternative strategy. An uncovered aortic dissection stent was placed into the aortic arch with the intention of stabilizing the aortic wall.

\section{CASE REPORT}

A 40-year-old male patient had brief but strong chest pain, as well as ongoing paresthesia and weakness of his whole right upper extremity after physical activity. He was taken to a rural hospital under emergency conditions, where a computed tomographic scan unveiled type A aortic dissection, also involving the aortic arch up to the abdominal aorta (Figure 1). The patient was immediately transferred by helicopter to our tertiary care institution to undergo surgery on an emergency basis. Extracorporeal circulation was connected through the right femoral artery and the right atrium because the right subclavian artery was heavily dissected and presented with an extensive vessel wall hematoma. The patient was cooled to $25^{\circ} \mathrm{C}$, and cardioplegic arrest was induced. Because the entry tear was found above the noncoronary sinotubular junction, reconstruction of the aortic root with French glue was simple. A $30-\mathrm{mm}$ Dacron polyester fabric prosthesis was anastomosed to the restored aortic root to replace the ascending aorta. After initiation of hypothermic circulatory arrest

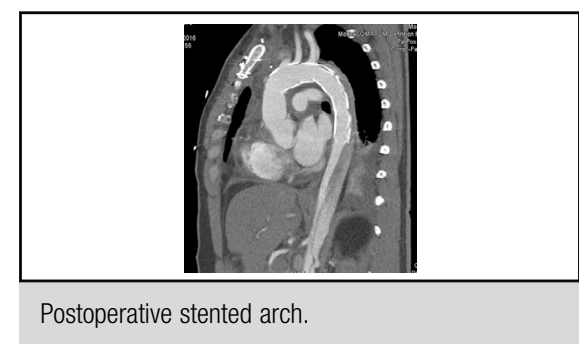

Central Message

Antegrade overstenting of the aortic arch with an uncovered stent to reverse true-lumen collapse in the setting of type A aortic dissection is feasible without a significant thromboembolic risk.

See Editorial Commentary page e201.

and selective antegrade cerebral perfusion through the ostia of the brachiocephalic trunk and the left carotid artery, inspection of the aortic arch did not reveal another endothelial laceration. The diameter of the proximal descending aorta was $32 \mathrm{~mm}$. The proximal arch was stabilized with French

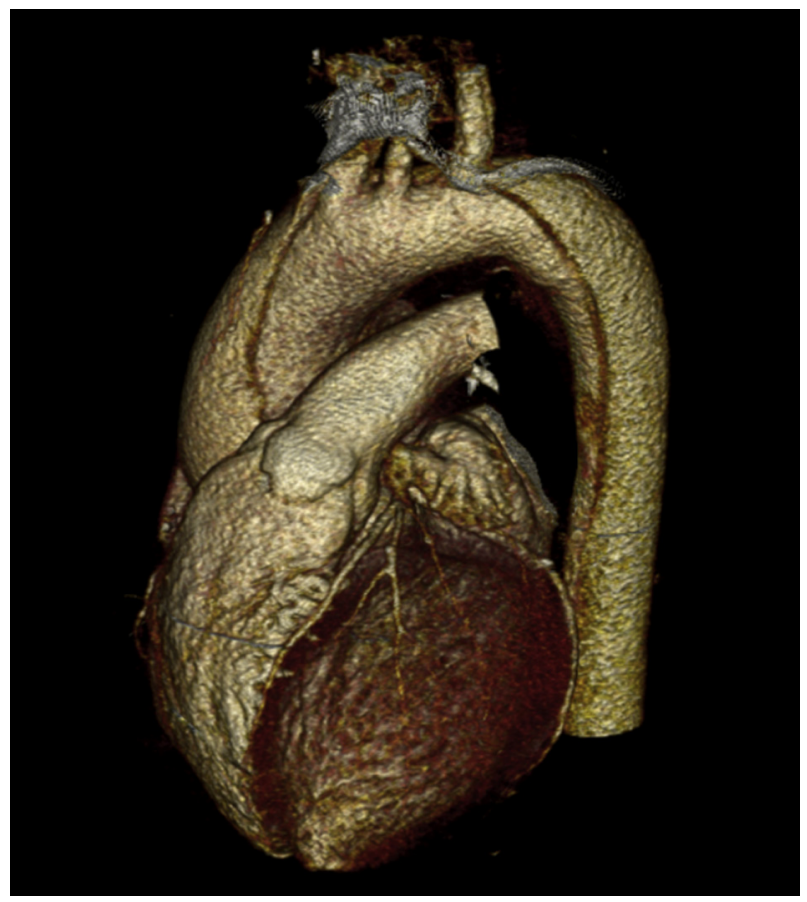

FIGURE 1. Preoperative 3-dimensional computed tomographic scan. 


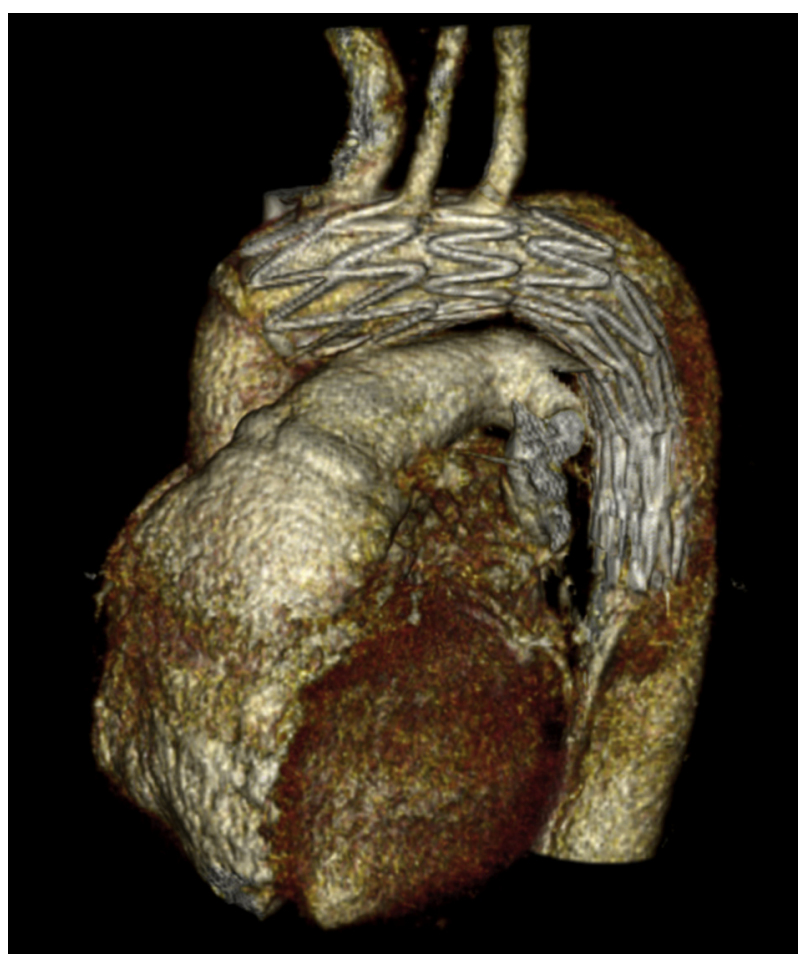

FIGURE 2. One-year postoperative 3-dimensional computed tomographic scan.

glue, and an uncovered $36 \mathrm{~mm} / 164 \mathrm{~mm}$ Cook aortic dissection stent (Cook Medical, Limerick, Ireland) was deployed in an antegrade fashion, overstenting all 3 supra-aortic vessels without the use of a guidewire. Before stent deployment, selective cerebral perfusion was terminated to avoid impingement of the catheters. The Dacron polyester fabric prosthesis was appropriately shortened and anastomosed end to end with the aortic arch and enclosed aortic stent. The operation was finished in a routine manner. The patient had a lifelong daily regimen of aspirin at $100 \mathrm{mg}$ prescribed.

\section{DISCUSSION}

Hybrid procedures combining stent placement and cardiovascular surgery are frequently considered a good solution to the quest for an optimal treatment strategy that avoids too-extensive surgery for type A aortic dissection with involvement of the aortic arch. Placing a covered stent beyond the offspring of the subclavian (or left carotid) artery may stabilize the aortic wall and reopen a so-called true-lumen collapse; however, this leaves the untreated arch at risk for later complications. ${ }^{2}$ To cope with this problem, the insertion of an uncovered stent into the aortic arch may serve as alternative treatment option. ${ }^{3,4}$ Although this certainly widens the true lumen, it is unclear whether thrombus formation and consecutive cerebral embolism may occur, and whether a false lumen can be successfully obliterated. Our patient was rather young and had preoperative paralysis, a protruding dissection membrane in the aortic arch, and a true-lumen collapse in the descending aorta. Extensive surgery was considered to carry an incalculable neurologic risk. Antegrade stent insertion during hypothermic circulatory arrest was therefore chosen. The exact stent placement through the open aortic arch proved somewhat tricky without fluoroscopy, because no markers were visible outside the covered stent, but it as finally successful. The postoperative computed tomographic scan demonstrated a reopened true lumen in the aortic arch and descending aorta; however, the false lumen was not obliterated. Obviously, the radial forces of the stents were not strong enough, assuming that the stent size was correct. A larger stent was considered too dangerous because of the possibility of penetrating the fragile vessel wall; however, there was no antegrade feeding of the false lumen, leaving open the option of inserting additional (covered) stents into the descending aorta. The immediate postoperative course was uneventful. The patient left the intensive care unit on postoperative day 2 and was discharged home 6 days later without neurologic symptoms. No rehabilitation was necessary. During the follow-up, no thromboembolic events occurred related to the stent. The 1-year control demonstrated identical findings (Figure 2).

In the future, this technique may also be used in uncomplicated cases. By now, a few research groups and companies have tried to develop an adequate stent to facilitate and hasten surgery for patients with acute type A dissection. Future prospective trials will show its value. The best indication will probably be a dissected aortic arch without an endothelial laceration. In the case of an entry tear in the aortic arch, arch replacement remains the better solution. In the case of aneurysmal descending thoracic artery, the stent should be fixed proximally with sutures to avoid displacement. Additional covered stents would then be added distally by interventional techniques to stop retrograde false-lumen perfusion.

In conclusion, placement of an uncovered stent during acute type A aortic dissection surgery to reopen a truelumen collapse is possible. Subsequent occlusion of the false lumen cannot be anticipated.

\section{References}

1. Kobuch R, Hilker M, Rupprecht L, Hirt S, Keyser A, Puehler T, et al. Late reoperations after repaired acute type A aortic dissection. J Thorac Cardiovasc Surg. 2012;144:300-7.

2. Sultan I, Wallen TJ, Habertheuer A, Siki M, Arnaoutakis GJ, Bavaria J, et al. Concomitant antegrade stent grafting of the descending thoracic aorta during transverse hemiarch reconstruction for acute DeBakey I aortic dissection repair improves aortic remodeling. J Card Surg. 2017;32:581-92.

3. Pasic M, Zipfel B, Drews T, Dreysse S, Unbehaun A, Buz S, et al. Transapical placement of an uncovered aortic endostent for type A aortic dissection. Circ Cardiovasc Interv. 2011;4:e49-53.

4. Massmann A, Kunihara T, Fries P, Schneider G, Buecker A, Schäfers HJ. Uncovered stent implantation in complicated acute aortic dissection type B. J Thorac Cardiovasc Surg. 2014;148:3003-11. 